\title{
The Application of Carbon Nanotubes in Magnetic Fluid Hyperthermia
}

\author{
Grzegorz Raniszewski, ${ }^{1}$ Arkadiusz Miaskowski, ${ }^{2}$ and Slawomir Wiak1 \\ ${ }^{1}$ Lodz University of Technology, Stefanowskiego Street 18/22, 90-924 Lodz, Poland \\ ${ }^{2}$ University of Life Sciences in Lublin, Akademicka Street 13, 20-033 Lublin, Poland \\ Correspondence should be addressed to Grzegorz Raniszewski; grzegorz.raniszewski@p.lodz.pl
}

Received 22 May 2015; Revised 12 July 2015; Accepted 14 July 2015

Academic Editor: Anh-Tuan Le

Copyright (c) 2015 Grzegorz Raniszewski et al. This is an open access article distributed under the Creative Commons Attribution License, which permits unrestricted use, distribution, and reproduction in any medium, provided the original work is properly cited.

\begin{abstract}
The aim of this paper is to present the results of the investigation into the applications of carbon nanotubes with ferromagnetic nanoparticles as nanoheaters for targeted thermal ablation of cancer cells. Relevant nanoparticles' characteristics were exploited in terms of their functionality for biomedical applications and their magnetic properties were examined to determine heat generation efficiency induced by the exposure of the particles to an alternating magnetic field. The influence of the electromagnetic field on the human body tissues was assessed, providing quantitative measures of the interaction. The behavior of a liquid containing magnetic particles, during the exposure to the alternating magnetic field, was verified. As for the application for the ferromagnetic carbon nanotubes, the authors investigated temperature distribution in human liver tumor together with Arrhenius tissue damage model and the thermal dose concept.
\end{abstract}

\section{Introduction}

The magnetic fluid hyperthermia (MFH) is a new and promising therapeutic technique. It works by increasing the temperature in the area of tumor, which triggers the natural mechanisms of self-destruction of the cells called apoptosis. Classical methods of hyperthermia suffer serious problems related to focusing the temperature rise at tumor area, and thus they cause side effects for the patient. In case of the MFH, it is different because the heating energy is provided directly to the target region. This is done by means of magnetic nanoparticles that are placed in the patient's body. When the nanoparticles are exposed to an alternating magnetic field with properly chosen frequency, the superparamagnetic heat phenomenon is observed.

Authors have assumed that a magnetic fluid with known ferromagnetic carbon nanotubes concentration was delivered directly to a tumor. It can be done when the location and extent of the tumor are known and it is easily accessible for delivery, for example, through an injection. Moreover, it is assumed that magnetic nanoparticles, together with tumor region, create homogeneous composite and their physical parameters are expressed in the form of effective ones. Moreover, we have not considered the power density due to the eddy currents as they are negligible regarding the heating of the injected magnetic fluid. On the other hand, the authors are conscious that eddy currents are important when talking about inductive heating. The maximum power density values in the body are determined by the size of the body, and so is the distance between the applicator and the skin. But this phenomenon will not be discussed in this study.

\section{Synthesis of Ferromagnetic Carbon Nanotubes}

There exists an extensive research on magnetic nanoparticles (MNPs) heated in a magnetic field as a tool for destroying cancer [1-6]. Among different kinds of MNPs, carbon nanotubes have gained interest of scientists due to very promising 


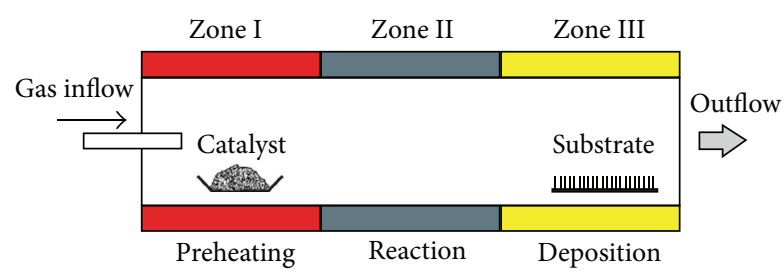

FIGURE 1: The experimental setup.

physical properties. The nanotubes have the advantage over other MNPs. Their quasi-one-dimensional shape renders them more efficient in terms of heat generation due to higher coercivity and saturation of magnetization of the CNTs as compared to spherical particles $[7,8]$.

The most common methods for carbon nanotubes (CNTs) synthesis are various types of chemical vapor deposition (CVD) and arc discharge methods. In CVD methods, gases containing carbon are usually used as a source of carbon for CNTs formation. In arc discharge methods, carbon comes from graphite anode. In the arc reactors, two types of carbon nanotubes can be collected: single-wall carbon nanotubes (SWNTs) and multiwall carbon nanotubes (MWNTs). MWNTs are usually located inside the cathode deposit. The syntheses of SWNTs need application of catalysts and they are deposited on the reactor walls, especially on the top of the reactor and in the anode area.

In CVD methods, the carbon for formation of carbon nanotubes is delivered in gaseous form. Two most common methods of carbon introduction into the system are application of hydrocarbons such as ethylene and acetylene. Gases with carbon gas decompose in high temperature and form carbon structures such as carbon nanotubes. Another group of CVD systems employ catalysts with carbons in solid or liquid state. Catalysts vaporize and are deposited as carbon nanotubes on substrates.

\section{An Experiment}

The method used for ferromagnetic carbon nanotubes synthesis was chemical vapor deposition with the tube furnace. As the carbon source ethylene was chosen, ferrocene was used as the catalyst. In the experimental setup, shown in Figure 1, four gases such as argon, helium, hydrogen, and ethylene were used and introduced into the furnace which was divided into three zones. In the first zone, inside the quartz tube, there was placed a catalyst in a ceramic boat. Relatively low temperature of this zone $(500 \mathrm{~K})$ allows for slowing evaporation of catalyst containing iron and carbon. The second zone was for regulation of the reaction time $(500-1000 \mathrm{~K})$. Too low temperature caused lack of catalyst decomposition. In the third zone, there was a silicon plate, substrate on which the CNTs should be deposited. The temperature of the third zone was about $1000 \mathrm{~K}$. These parameters allowed for formation of carbon nanotubes with iron nanoparticles (Fe-CNTs). The scheme of Fe-CNT's formation is presented in Figure 2.

Carbon nanotubes collected from the substrate (and from the quartz tube walls) are contaminated with soot and

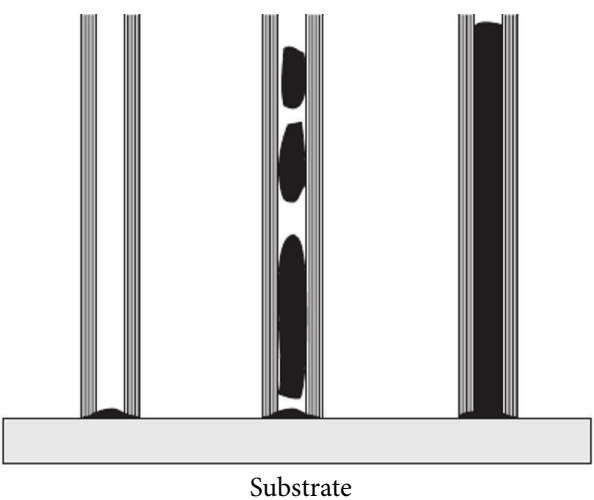

FIGURE 2: Carbon nanotubes filled with iron.

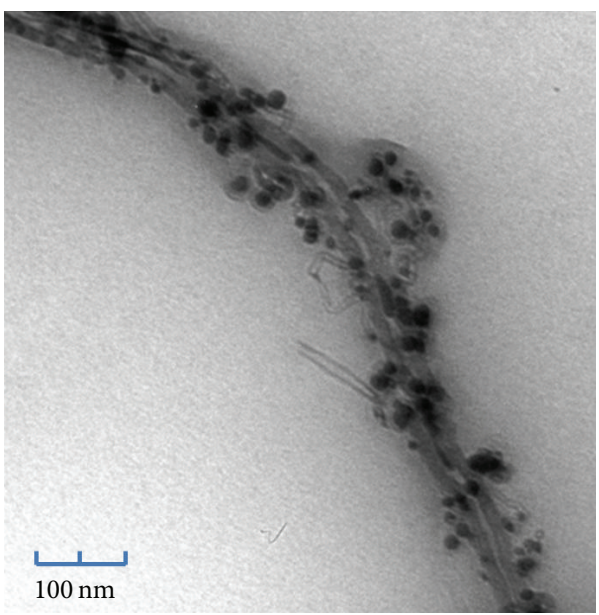

Figure 3: TEM of ferromagnetic nanotubes.

amorphous carbon. To purify the final product, an oxidation in high temperature $(700 \mathrm{~K})$ was used. Carbon impurities oxidize in low temperatures. Carbon nanotubes due to their thermal properties are resistant to temperatures up to $1000 \mathrm{~K}$ [11].

\section{Results}

Carbon nanotubes produced by this method were characterized by their ability to generate heat caused by the electromagnetic field with known parameters, that is, frequency and magnetic flux density. Moreover, to investigate if the thermal oxidation was an appropriate method of purification, thermogravimetric test was done. The iron contamination in the sample was estimated by EDS analysis. It was found that $5-15 \mathrm{wt} \%$ of iron was found in CNTs synthesized at temperatures $1000-1100 \mathrm{~K}$. The iron spontaneously filled carbon nanotubes in different ratios. Some of the iron nanoparticles were attached to the surface outside the nanotubes (Figure 3).

Although fulfillment of the nanotubes has a much larger volume, the majority of molecules placed outside the nanotube have the size of nanometers. It was calculated that average diameter of iron nanoparticles was $10.3 \mathrm{~nm}$. To estimate the diameter of nanotubes, dynamic light scattering (DLS) 
has been used. Multiwalled carbon nanotubes synthesized by this method have length around $30-50 \mu \mathrm{m}$ and diameter of $30-60 \mathrm{~nm}$.

To determine the cytotoxicity ferromagnetic carbon nanotubes and their effects on cell, viability tests on the cell lines were conducted. A variety of carbon nanotubes concentration in the culture cell medium was prepared in the range of 5$500 \mathrm{mg} / \mathrm{L}$. There was no significant influence of CNTs on cell viability. It indicates that they are inert to the cells. After functionalization (ligands conjugation to the carbon nanotubes structure) and attachment to the cells, they will have a selective destructive effect on cancer cells in the electromagnetic field.

The interaction of magnetic nanoparticles with the electromagnetic field depends on the particles' properties, their morphology, and suspending medium (if present), as well as the field parameters. Combining all the above factors, various magnetic loss mechanisms can be distinguished. Knowing the mechanism, the quantity of heat generated by a certain amount of particles can be calculated per one period of oscillating field. The quantity is generally referred to as Specific Magnetic Loss Energy (SMLE). In order to obtain the overall heating capacity of a given particle amount in a specific field, the Specific Loss Power (SLP) is calculated, by multiplying SMLE with the frequency $f$ of the field applied. In different magnetic loss mechanisms, the SLP is estimated using various relations.

Ferromagnetic-doped CNTs in the form of powder produce heat when exposed to an alternating magnetic field. The atomic dipoles' alignment, as a response to the magnetic field, can be represented by a hysteretic dependence of magnetization $(\mathrm{M})$ versus magnetic field strength $(\mathrm{H})$. The heat generation is a result of hysteresis losses due to the reversing of magnetization [12-14].

Ferromagnetic nanoparticles generate heat in cases when an applied magnetic field is stronger than the coercivity that is applied. This is the reason why the low coercivity nanoparticles are preferred. Ferromagnetic carbon nanotubes are able to generate heat with the application of low magnetic fields [15]. In hyperthermia applications, the applied magnetic field ranges from $10 \mathrm{mT}$ to about $25 \mathrm{mT}$ at a frequency of $\mathrm{kHz}$. The high frequency is required to avoid pain. The small field will only partially magnetize the nanoparticles. In $\mathrm{kHz}$ frequencies, AC loss effects are also significant. Figure 4 shows the hysteresis loops for the sample obtained from CVD system for temperature $300 \mathrm{~K}$. They are compared to the hysteresis loops for the sample without iron (Figure 5). As a reference sample, Nanocyl NC 7000 has been used. As expected, the carbon nanotubes with ferromagnetic nanoparticles show a significant hysteresis compared to ferromagnetic properties of multiwall carbon nanotubes from the market.

The amount of heat generated by the magnetic nanoparticles is commonly expressed in terms of the SAR, which is understood as the power generated per unit mass of magnetic material in the solution. SAR is given by

$$
\mathrm{SAR}=c \cdot \frac{m_{\text {sample }}}{m_{\mathrm{Fe}}} \frac{\Delta T}{\Delta t},
$$

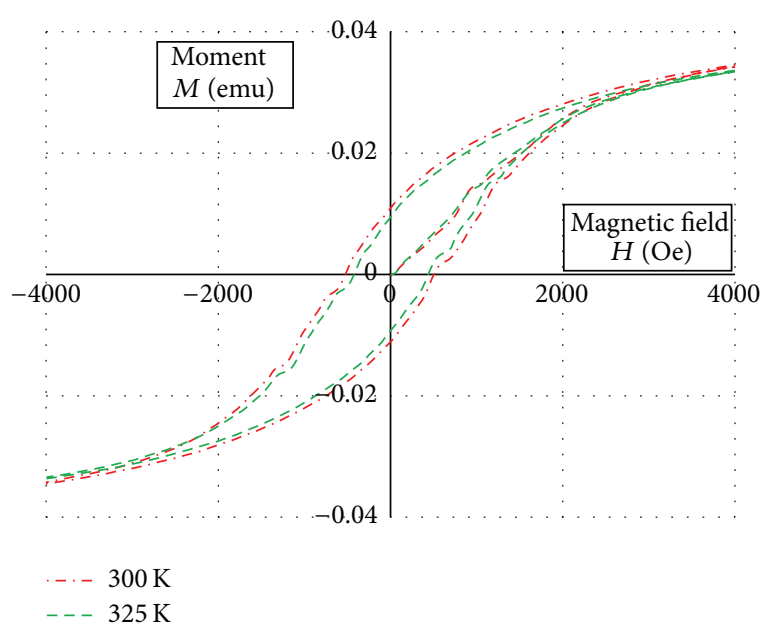

FIGURE 4: Hysteresis loop for carbon nanotubes with ferromagnetic nanoparticles.

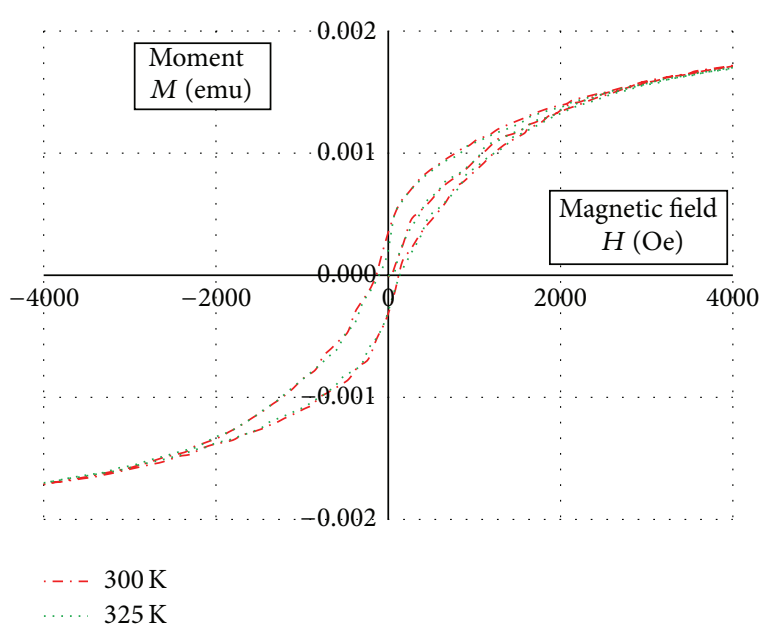

FIGURE 5: Hysteresis loop for carbon nanotubes without ferromagnetic nanoparticles.

where $c$ is the heat capacity of the colloid, $m_{\text {sample }}$ is the mass of the sample, $m_{\mathrm{Fe}}$ is the mas of iron oxide in the sample, and $\Delta T / \Delta t$ represents the heating rate. The volumetric energy dissipation rates $Q_{\text {ext }}$ and SAR are related by

$$
Q_{\mathrm{ext}}=\mathrm{SAR} \cdot w_{\mathrm{Fe}}=\mathrm{SAR} \cdot \varphi \cdot \rho_{\mathrm{Fe}},
$$

where $w_{\mathrm{Fe}}$ is the mass of iron oxide per unit volume, $\varphi$ is the volume fraction on iron oxide, and $\rho_{\mathrm{Fe}}$ is the density of iron oxide. It is very important to note that (2) is accurate if the heat capacity of the nanoparticles is in thermal equilibrium with its surroundings at the start of the experiments. Otherwise, heat flow to/from the environment can confound the measurements [16].

On the other hand, the SAR parameter for the same magnetic fluid will change when measured in AC magnetic field systems with different frequencies and strengths, as 


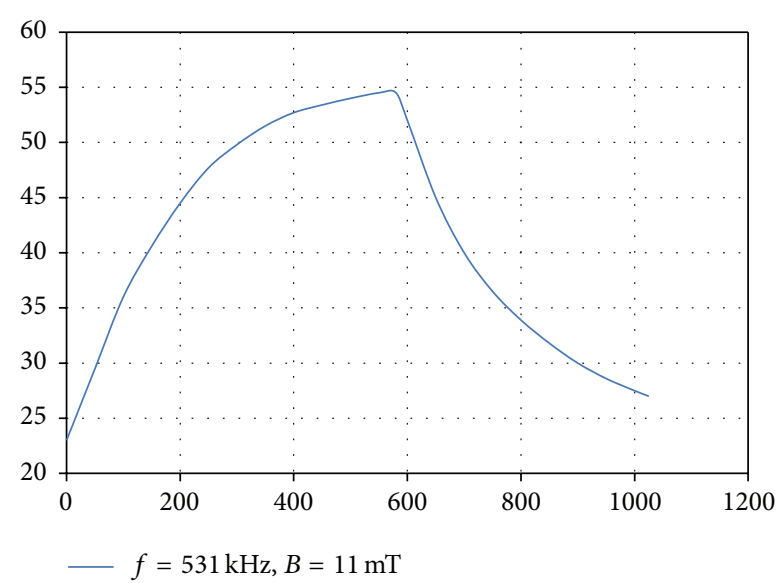

FIGURE 6: The heating profile of $2 \mathrm{~mL}$ DMSA stabilized magnetite carbon nanotubes dispersed in water, $B=11 \mathrm{mT}, f=532 \mathrm{kHz}$.

can be deduced from the formula for volumetric power dissipation [5]:

$$
Q_{\text {ext }}=\mu_{0} \pi \chi^{\prime \prime} f H_{0}^{2}
$$

where $\mu_{0}$ is the permeability of free space, $\chi$ is the out-ofphase (imaginary) component of susceptibility, and $H$ is the applied magnetic field strength.

\section{CNT's Dispersion in Aqueous Media}

In our case, the magnetic fluid was prepared by ferromagnetic carbon nanotubes dispersion in aqueous media. Due to their insolubility in any sort of solvents resulting from strong Van der Waals forces, extremely high aspect ratio, and flexibility of the particles, the particles tend to form bundles or agglomerates [17-19]. The dispersion is achieved by ultrasonication.

The examined $\mathrm{Fe}$ concentrations were from 5 to $75 \mathrm{mg} / \mathrm{mL}$, whereas the average magnetic particle diameter was $10.3 \mathrm{~nm}$. Moreover, ferromagnetic nanotubes were DMSA stabilized and dispersed in distilled water. The heating properties of the ferrofluid were measured using Nanotherics Magnetherm system. The system consists of a function generator and a power amplifier and it is monitored by oscilloscope. During measurements, the sample was placed in a coil enclosure and the temperature was measured with a fiberoptic temperature sensor. The data collected by the sensor are shown in Figure 6 at frequency $f=530 \mathrm{kHz}$.

In order to validate the measured data, the numerical investigation was prepared and the results of both the numerical and the experimental investigations were compared. The numerical model of the Magnetherm system was prepared using Semcad X software (Figure 7). During the experiments, the 9-turn coil leading current $I_{\text {rms }}=43[\mathrm{~A}]$ was used. The numerical axisymmetric model of the $40 \mathrm{~mm}$ diameter copper's coil can be seen in Figure 8.

To calculate a magnetic flux distribution in the coil, the low frequency solver was applied. In our case, we have used the low frequency magnetoquasistatic algorithm where the electric field $\mathbf{E}$ is calculated in the lossy $(\sigma \neq 0)$

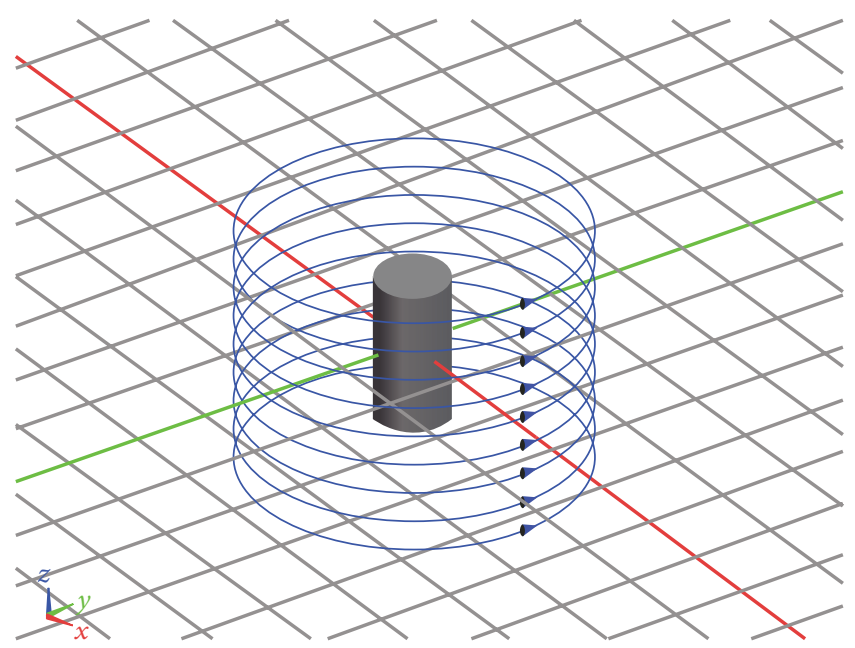

FIGURE 7: The numerical model of 9-turn coil used in the system with the ferrofluid sample.

domain, whereas the magnetic field $\mathbf{H}$ is calculated overall. The magnetic flux distribution in the middle cross-section can be seen in Figure 9. It can be seen that the sample is exposed to a uniform magnetic field and it can be assumed that the sample is uniformly heated.

\section{Temperature Distribution in Biological Cell Hyperthermia Cancer Treatment}

6.1. Thermal Problem. For heat transfer in biological tissues in this study, Pennes' bioheat transfer equation was used [20]:

$$
\rho c \frac{\partial T}{\partial t}=\nabla \cdot(\kappa \nabla T)-\rho_{b} c_{b} \omega\left(T-T_{b}\right)+Q_{\text {met }}+Q_{\text {ext }},
$$

where $\rho$ is the tissue density, $c$ is tissue specific heat, $\rho_{b}$ is the density of blood, $c_{b}$ is blood specific heat, $\kappa$ is tissue thermal conductivity, $\omega$ is blood perfusion rate, $T_{b}$ is arterial blood temperature, and $Q_{\text {met }}$ is metabolic heat source. The cooling associated with the heat exchange with environment was imposed in the form of Robin's boundary condition; that is,

$$
\kappa \frac{\partial T}{\partial n}=h\left(T_{\mathrm{ext}}-T\right),
$$

where $h$ is the heat transfer coefficient and $T_{\text {ext }}$ is the external temperature.

In the case of magnetic fluid hyperthermia, the $Q_{\text {ext }}$ term is the power density (the energy deposition) due to heating of the magnetic fluid in an AC magnetic field. In this study, the authors have used the SAR value received from experiments to numerically investigate a temperature distribution in the anatomically correct human model. A special attention has been paid on the model of the tumor region with magnetic fluid, where the values for the electrical conductivity $\sigma$, the density $\rho$, the heat capacity $c$, and the thermal conductivity $\kappa$ have been calculated as the effective one as follows:

$$
\rho=(1-\varphi) \rho_{\text {tumor }}+\varphi \rho_{\mathrm{MF}} \text {. }
$$




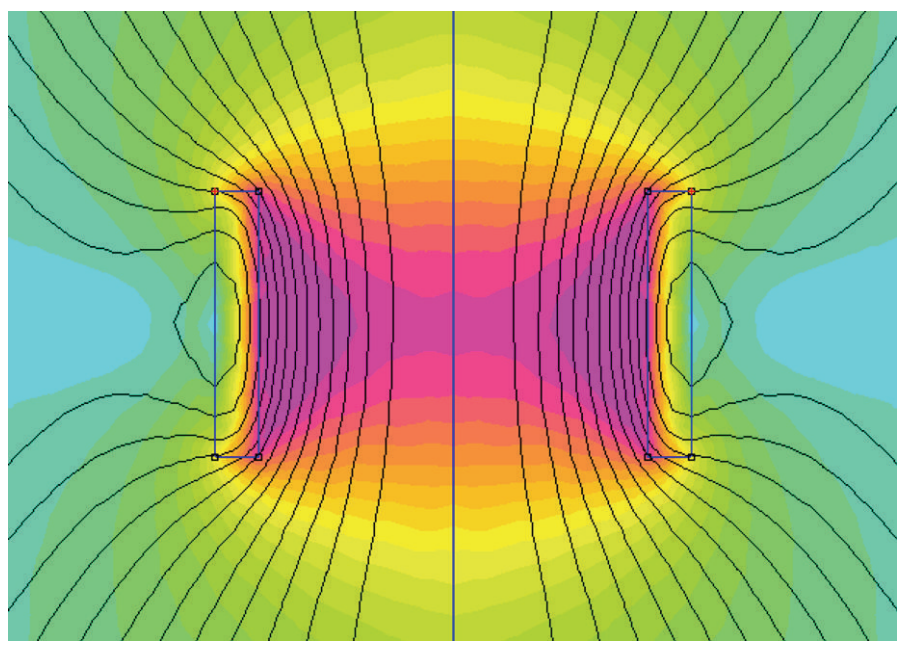

Figure 8: The magnetic flux distribution in the 9-turn coil used in the experiments.

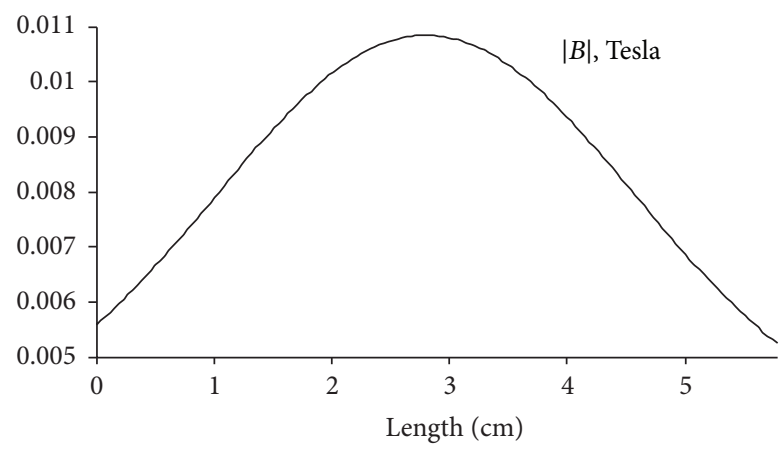

FIgURE 9: Magnetic flux distribution along $z$-axis

The effective specific heat is as follows:

$$
c=(1-\varphi) c_{\text {tumor }}+\varphi c_{\mathrm{MF}} .
$$

The effective thermal conductivity is as follows:

$$
\frac{1}{\kappa}=\frac{1-\varphi}{\kappa_{\text {tumor }}}+\frac{\varphi}{\kappa_{\mathrm{MF}}}
$$

and the effective electrical conductivity is as follows:

$$
\frac{1}{\sigma}=\frac{1-\varphi}{\sigma_{\text {tumor }}}+\frac{\varphi}{\sigma_{\mathrm{MF}}},
$$

where subscript MF indicates magnetic fluid. That means that the tumor region was treated as a homogeneous composite. Moreover, a blood heat capacity $c_{b}=4.05 \cdot 10^{6}$ and liver metabolic heat $Q_{\text {met }}=11.37 \mathrm{~W} / \mathrm{m}^{3}$ were used in the Pennes model.

The temperature distribution in the human tissues is determined by a number of thermophysical factors, such as heat capacity and thermal conductivity, the spatial geometry (anatomy), and heat production due to metabolism. There are high-resolution numerical phantoms based MRI imagines

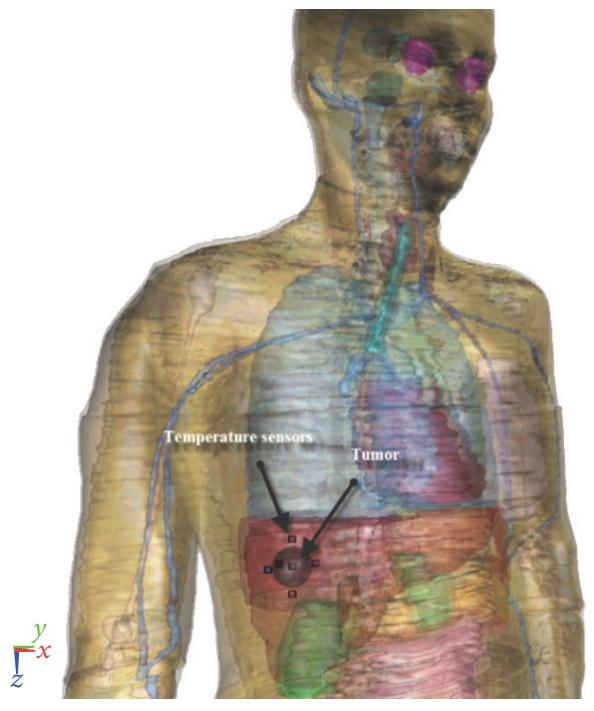

FIGURE 10: The model of Ella with liver tumor and points in which the temperature was measured.

which can be used to predict thermophysical effects. In this work, the authors have used an anatomically correct model from Virtual Population [21]. The model represents a 26-yearold female, with $1.63 \mathrm{~m}$ height and $58.7 \mathrm{~kg}$ weight (Figure 10). To numerically model a liver tumor, the sphere with radius $r=15 \mathrm{~mm}$ has been embedded in it. The properties of tissues were taken, obtained from the database of the IT'IS Foundation [22] and the calculations was fixed at a frequency of $530 \mathrm{kHz}$. The liver tumor electric and thermal properties were taken from the literature $[9,10]$ (see Table 1). In our case, the $\mathrm{SAR}=41.8 \mathrm{~W} / \mathrm{kg}$.

6.2. Tissue Damage Model. Apart from the temperature distribution, the Arrhenius model was used to estimate tissue damage as another parameter. In this model, it is assumed 


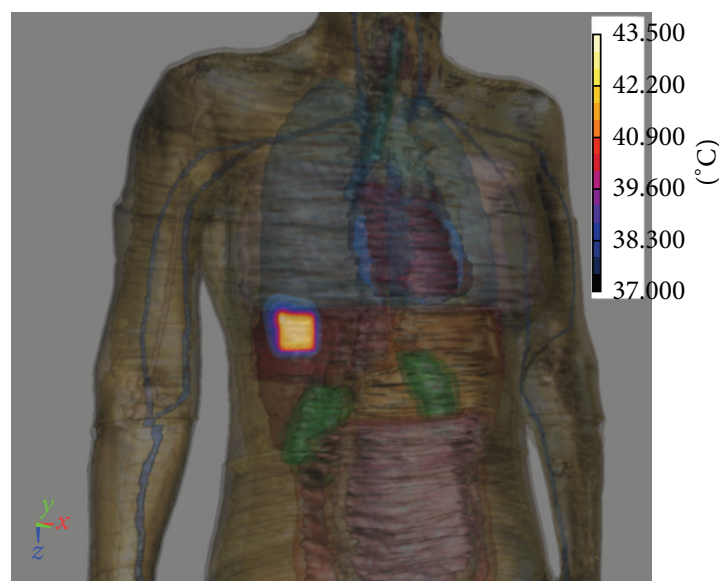

(a)

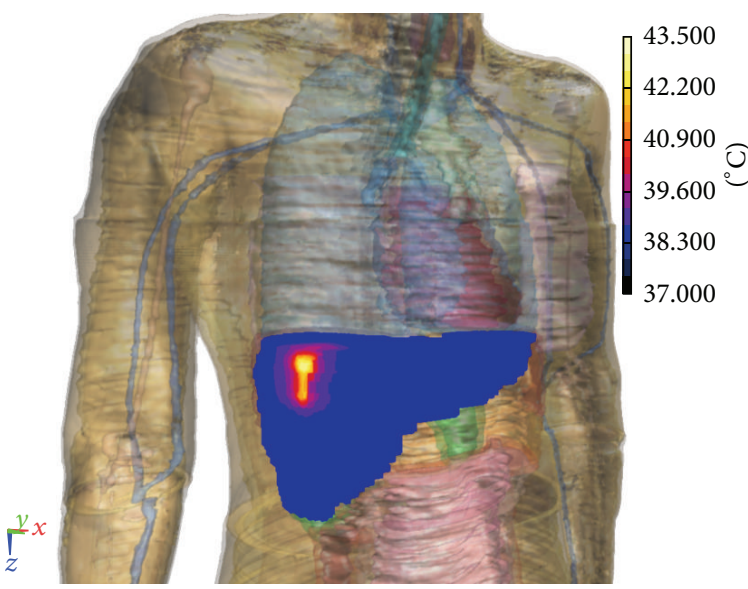

(b)

FIGURE 11: Temperature distribution in the liver tumor after 1800 sek. (cross-section (a), surface temperature distribution (b)).

TABLE 1: Electric and thermal properties of healthy liver and the one with tumor $[9,10]$.

\begin{tabular}{lccccc}
\hline Tissue & $\rho\left(\mathrm{kg} \cdot \mathrm{m}^{-3}\right)$ & $C\left(\mathrm{~J} \cdot \mathrm{kg}^{-1} \mathrm{~K}^{-1}\right)$ & $\kappa\left(\mathrm{W} \cdot \mathrm{m}^{-1} \mathrm{~K}^{-1}\right)$ & $\sigma\left(\mathrm{S} \cdot \mathrm{m}^{-1}\right)$ & $\omega_{0}\left(\mathrm{~kg} \cdot \mathrm{m}^{-3} \mathrm{~s}^{-1}\right)$ \\
\hline Healthy liver & 1080 & 3455 & 0.173 & 0.51 & 17.03 \\
\hline Liver tumor & 1045 & 3760 & 0.52 & 0.5 & 2.09 \\
\hline
\end{tabular}

TABLE 2: Kinetic coefficients for the healthy liver and liver tumor $[9,10]$.

\begin{tabular}{lcc}
\hline Tissue & $A\left(\mathrm{~s}^{-1}\right)$ & $\Delta E(\mathrm{~J} / \mathrm{mol})$ \\
\hline Healthy liver & $7.390 \cdot 10^{39}$ & $2.577 \cdot 10^{5}$ \\
\hline Liver tumor & $3.247 \cdot 10^{43}$ & $2.814 \cdot 10^{5}$ \\
\hline
\end{tabular}

that the biological damage depends on both temperature and time and can be quantified by the function of tissue injury $\Omega$ :

$$
\Omega(t)=\int_{0}^{t} A e^{-\Delta E / R T} d t
$$

where $T$ is the absolute temperature, which is a function of ablation time, $R$ is the gas constant $8.314 \mathrm{~J} /(\mathrm{mol} \mathrm{K})), A$ is the frequency factor $\left(s^{-1}\right)$, and $\Delta E$ is the energy activation for the irreversible damage reaction. Kinetic coefficients $A$ and $\Delta E$ are presented in Table 2 . It should be underlined that $\Omega(t)$ is the damage integral, while $F=1-e^{-\Omega(t)}$ and corresponds to a $63 \%$ probability of the death of cells if $\Omega(t)=1$. In other words, at $\Omega(t)<1$, the tissue is considered as a live one.

6.3. Thermal Dose. Together with the temperature distribution and Arrhenius tissue damage model, the authors have analyzed the thermal factor, which is the thermal dose concept developed by Sapareto and Dewey in 1984 [23]. Cumulative equivalent minutes at $43^{\circ} \mathrm{C}\left(\mathrm{CEM}_{43}\right)$ are defined as

$$
\mathrm{CEM}_{43}=\int_{0}^{t} R^{43-T(t)} d t
$$

with $R=0.5$ for $T>43, R=0.25$ for $43 \geq T \geq 39$, and $R=0$ for $T<39$.
When the $\mathrm{CEM}_{43}$ value exceeds a critical threshold, the tissue is considered to be thermally necrosed. Thresholds for $\mathrm{CEM}_{43}$ have been tabulated in the literature for several types of tissue based upon experimental observation of tissue injury at $43^{\circ} \mathrm{C}$ [24-28].

The critical thermal dose for liver tissue is $\mathrm{CEM}_{43}=$ 340 minutes [29]. Once the temperature was determined at each time step, the $\mathrm{CEM}_{43}$ was calculated. The tissue was considered to begin necrosis when this factor exceeded 40 minutes [30], although significant degree of damage appears when cumulative equivalent is not less than 240 minutes [3133].

\section{Results}

Figure 11 shows the temperature distribution in the liver tumor after $1800 \mathrm{sec}$. The important observation in Figure 11 is the temporal evaluation of temperature at various points which is unsymmetrical. The effect of anatomy (the liver shape) can be seen in Figure 11 where it is shown that the maximum temperature is reached at the different locations, which may have an implication for expected treatment planning.

The cumulative equivalent minutes for this case are shown in Figure 12.

It can be observed that maximum temperature $T=$ $43.5^{\circ} \mathrm{C}$ in the middle of the tumor was reached after about 300 sek. (Figure 13). In Figure 14, there are temperature profiles in the thermosensors symmetrically surrounding the tumor. In our numerical experiments, it was assumed that magnetic nanoparticles were dispersed homogeneously, but the temperatures on the tumor boundary did not have the same profiles. One should notice that the liver is surrounded 


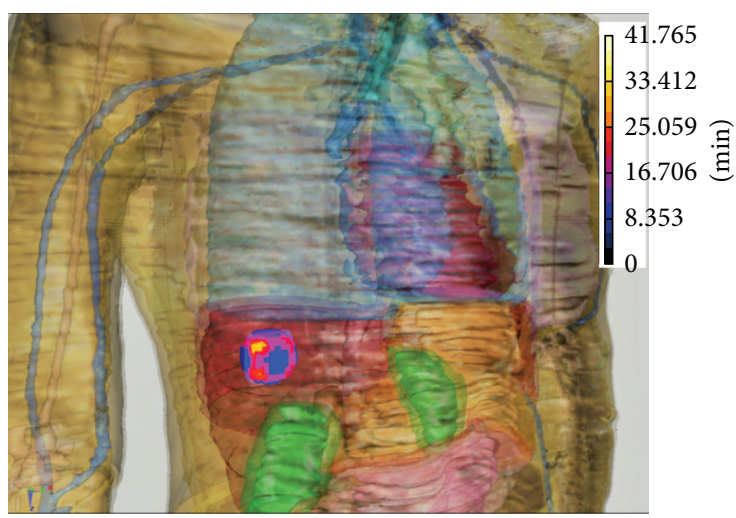

Figure 12: $\mathrm{CEM}_{43}$.

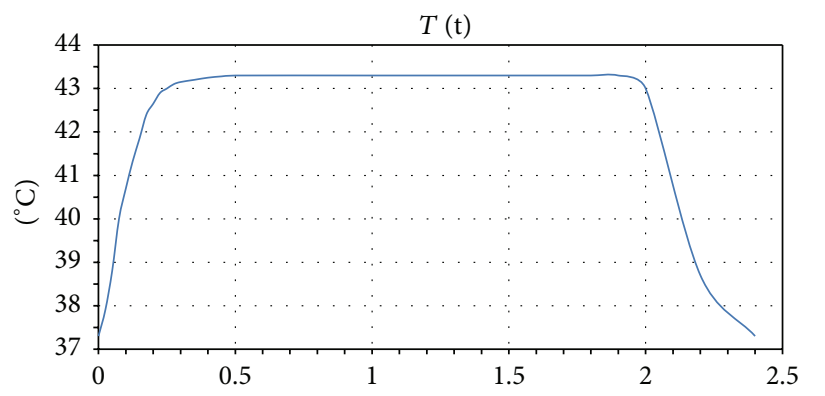

(ks)

— Thermosimulation 1, thermopoint sensor 1

Figure 13: Temperature versus time for different thermal sensors, sensor placed in the middle of the tumor.

by different tissues and due to different perfusion rates the tumor boundaries will be cooled with various rates.

\section{Conclusions}

There are many methods of carbon nanotubes synthesis. The main methods employ high temperature (e.g., arc discharge method) and low temperature (e.g., chemical vapor deposition). Although both methods need purification of the results of synthesis, CVD method is preferred in this study. In our research, we used a ferrocene as a catalyst and to fill carbon nanotubes by iron. These carbon nanotubes work then as ferrofluids but in contrast to ferrofluids CNTs may be easily biofunctionalized and then can be introduced into the human body. Cancer cells overexpress folic acid (FA) as well as epidermal growth factor (EGF) receptors. Carbon nanotubes with FA and EGF show preference for binding to a cancer cell which is an advantage in relation to ferromagnetic fluids. Application of carbon nanotubes with ferromagnetic properties can be used in a radiofrequency thermal ablation. RF ablation therapy brings the lowest risk compared to the other techniques (e.g., surgery, chemotherapy) of tumor therapy. This method offers faster and more targeted treatment for liver cancer with fewer side effects. The absorption coefficient of radio waves by the human body is relatively low. Metals in the carbon nanotube absorb this energy much

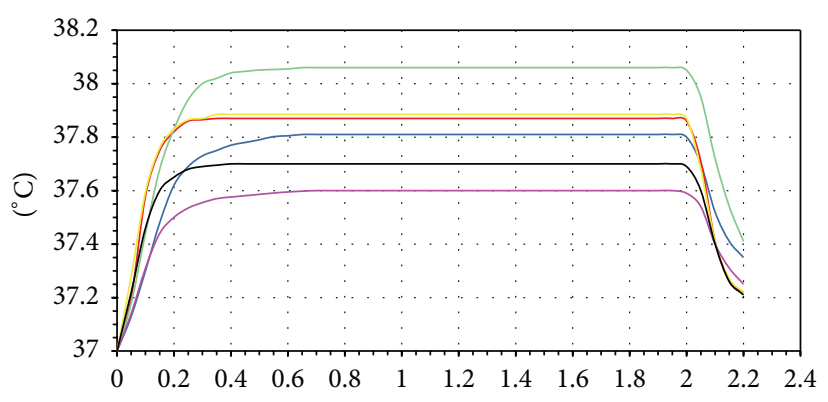

(ks)

Thermosimulation 1 , thermopoint sensor 2
Thermosimulation 1 , thermopoint sensor 3
Thermosimulation 1 , thermopoint sensor 4
Thermosimulation 1 , thermopoint sensor 5
Thermosimulation 1 , thermopoint sensor 6
Thermosimulation 1 , thermopoint sensor 7

FIgURE 14: Temperature versus time for different thermal sensors, sensors placed on the tumor boundary.

more efficiently than a tissue. In this paper, prediction of spatial temperature distribution in the anatomically correct human model has been presented. Based on experimental and numerical investigation, the power dissipation from ferromagnetic carbon nanotubes has been investigated. The authors have shown that the carbon nanotubes have the potential for the hyperthermia treatment.

From the numerical investigation presented above, one can conclude that, in terms of maximum temperature rise, the expected treatment outcome depends on the tissue distribution around the tumor.

\section{Conflict of Interests}

The authors declare that there is no conflict of interests regarding the publication of this paper.

\section{Acknowledgment}

The research work is related to $\mathrm{R} \& \mathrm{D}$ Grant and $\mathrm{Nr}$ PBS2/A5/31/2013.

\section{References}

[1] J. Carrey, B. Mehdaoui, and M. Respaud, "Simple models for dynamic hysteresis loopcalculations of magnetic singledomain nanoparticles: application to magnetic hyperthermia optimization," Journal of Applied Physics, vol. 109, Article ID 083921, 2011.

[2] G. Vallejo-Fernandez, O. Whear, A. G. Roca et al., "Mechanisms of hyperthermia in magnetic nanoparticles," Journal of Physics D: Applied Physics, vol. 46, no. 31, Article ID 312001, 2013.

[3] H. Nemala, J. S. Thakur, V. M. Naik, P. P. Vaishnava, G. Lawes, and R. Naik, "Investigation of magnetic properties of $\mathrm{Fe}_{3} \mathrm{O}_{4}$ nanoparticles using temperature dependent magnetic hyperthermia in ferrofluids," Journal of Applied Physics, vol. 116, no. 3, Article ID 034309, 2014. 
[4] P. Di Barba, F. Dughiero, and E. Sieni, "Magnetic field synthesis in the design of inductors for magnetic fluid hyperthermia," IEEE Transactions on Magnetics, vol. 46, no. 8, pp. 2931-2934, 2010.

[5] R. E. Rosensweig, "Heating magnetic fluid with alternating magnetic field," Journal of Magnetism and Magnetic Materials, vol. 252, no. 1-3, pp. 370-374, 2002.

[6] A. Miaskowski, B. Sawicki, and A. Krawczyk, "The use of magnetic nanoparticles in low frequency inductive hyperthermi," COMPEL - The International Journal for Computation and Mathematics in Electrical and Electronic Engineering, vol. 31, no. 3, pp. 1096-1104, 2012.

[7] G. Zhou, W. Duan, and B. Gu, "First-principles study on morphology and mechanical properties of single-walled carbon nanotube," Chemical Physics Letters, vol. 333, no. 5, pp. 344-349, 2001.

[8] E. S. Glazer and S. A. Curley, "Non-invasive radiofrequency ablation of malignancies mediated by quantum dots, gold nanoparticles and carbon nanotubes," Therapeutic Delivery, vol. 2, no. 10, pp. 1325-1330, 2011.

[9] B. Zhang, M. A. J. Moser, E. M. Zhang, Y. Luo, H. Zhang, and W. Zhang, "Study of the relationship between the target tissue necrosis volume and the target tissue size in liver tumours using two-compartment finite element RFA modelling," International Journal of Hyperthermia, vol. 30, no. 8, pp. 593-602, 2014.

[10] G. Zorbas and T. Samaras, "Simulation of radiofrequency ablation in real human anatomy," International Journal of Hyperthermia, vol. 30, no. 8, pp. 570-578, 2014.

[11] L. Szymanski, Electrothermal Synthesis of Carbon Nanotubes under Atmospheric Pressure, Zeszyty Naukowe Nr 1162, Lodz University of Technology Press: Scientific Bulletin of the Lodz University of Technology, Lodz, Poland, 2013, (Polish).

[12] K. Lipert, M. Ritschel, A. Leonhardt, Y. Krupskaya, B. Büchner, and R. Klingeler, "Magnetic properties of carbon nanotubes with and without catalyst," Journal of Physics: Conference Series, vol. 200, no. 7, Article ID 072061, 2010.

[13] U. Weissker, S. Hampel, A. Leonhardt, and B. Büchner, "Carbon nanotubes filled with ferromagnetic materials," Materials, vol. 3, no. 8, pp. 4387-4427, 2010.

[14] R. Burgess, Understanding Nanomedicine: An Introductory Textbook, Pan Stanford Pte, Singapore, 2012.

[15] T. Atsumi, B. Jeyadevan, Y. Sato, and K. Tohji, "Heating efficiency of magnetite particles exposed to AC magnetic field," Journal of Magnetism and Magnetic Materials, vol. 310, no. 2, pp. 2841-2843, 2007.

[16] B. Kozissnik, A. C. Bohorquez, J. Dobson, and C. Rinaldi, "Magnetic fluid hyperthermia: advances, challenges, and opportunity," International Journal of Hyperthermia, vol. 29, no. 8, pp. 706-714, 2013.

[17] J. Hilding, E. A. Grulke, Z. G. Zhang, and F. Lockwood, "Dispersion of carbon nanotubes in liquids," Journal of Dispersion Science and Technology, vol. 24, no. 1, pp. 1-41, 2003.

[18] A. Jorio and G. D. M. Dresselhaus, Carbon Nanotubes. Advanced Topics in the Synthesis, Structure, Properties and Applications, Springer, Berlin, Germany, 2008.

[19] Q. Cheng, S. Debnath, E. Gregan, and H. J. Byrne, "UltrasoundAssisted swnts dispersion: effects of sonication parameters and solvent properties," Journal of Physical Chemistry C, vol. 114, no. 19, pp. 8821-8827, 2010.

[20] H. H. Pennes, "Analysis of tissue and arterial blood temperatures in the resting human forearm," Journal of Applied Physiology, vol. 85, no. 1, pp. 5-34, 1998.
[21] 2015, http://www.itis.ethz.ch/itis-for-health/virtual-population/ overview/.

[22] P. A. Hasgall, F. Di Gennaro, C. Baumgartner et al., "IT'IS Database for thermal and electromagnetic parameters of biological tissues," Version 2.5, 2014, http://www.itis.ethz.ch/ database.

[23] S. A. Sapareto and W. C. Dewey, "Thermal dose determination in cancer therapy," International Journal of Radiation Oncology, Biology, Physics, vol. 10, no. 6, pp. 787-800, 1984.

[24] I. A. Chang and U. D. Nguyen, "Thermal modeling of lesion growth with radiofrequency ablation devices," BioMedical Engineering Online, vol. 3, article 27, 2004.

[25] S. A. Baldwin, A. Pelman, and J. L. Bert, "A heat transfer model of thermal balloon endometrial ablation," Annals of Biomedical Engineering, vol. 29, no. 11, pp. 1009-1018, 2001.

[26] H. H. Pennes, "Analysis of tissue and arterial blood temperature in the resting human forearm," Journal of Applied Physiology, vol. 1, pp. 93-122, 1948.

[27] M. J. Borelli, L. L. Thompson, C. A. Cain, and C. A. Dewey, "Time-temperature analysis of cell killing of BHK cells heated at temperatures in the range of $43.5^{\circ} \mathrm{C}$ to $57^{\circ} \mathrm{C}$, International Journal of Radiation Oncology, Biology, Physics, vol. 19, pp. 389399, 1990.

[28] P. S. Yarmolenko, E. J. Moon, C. Landon et al., "Thresholds for thermal damage to normal tissues: an update," International Journal of Hyperthermia, vol. 27, no. 4, pp. 320-343, 2011.

[29] S. J. Graham, L. Chen, M. Leitch et al., "Quantifying tissue damage due to focused ultrasound heating observed by MRI," Magnetic Resonance in Medicine, vol. 41, no. 2, pp. 321-328, 1999.

[30] M. R. Hemmila, D. S. Foley, A. V. Casetti, O. S. Soldes, R. B. Hirschl, and R. H. Bartlett, "Perfusion induced hyperthermia for oncologic therapy with cardiac and cerebral protection," ASAIO Journal, vol. 48, no. 4, pp. 350-354, 2002.

[31] O. Seror, M. Lepetit-Coiffé, B. Bail et al., "Real time monitoring of radiofrequency ablation based on MR thermometry and thermal dose in the pig liver in vivo," European Radiology, vol. 18, no. 2, pp. 408-416, 2008.

[32] M. Lepetit-Coiffé, B. Quesson, O. Seror et al., "Real-time monitoring of radiofrequency ablation of rabbit liver by respiratorygated quantitative temperature MRI," Journal of Magnetic Resonance Imaging, vol. 24, no. 1, pp. 152-159, 2006.

[33] J. Haveman, O. A. G. Smals, and H. M. Rodermond, "Effects of hyperthermia on the rat bladder: a pre-clinical a study on thermometry and functional damage after treatment," International Journal of Hyperthermia, vol. 19, no. 1, pp. 45-57, 2003. 

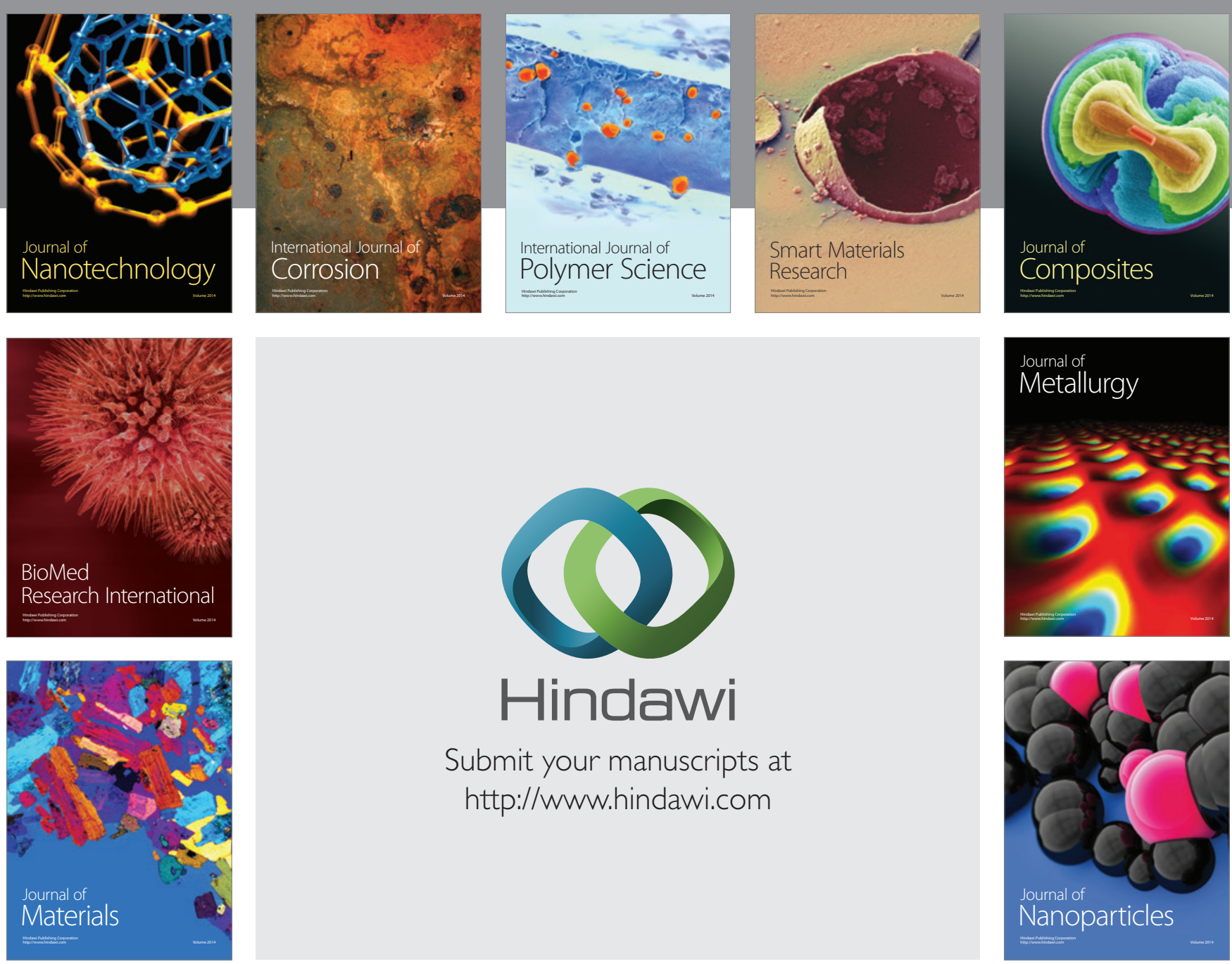

Submit your manuscripts at http://www.hindawi.com
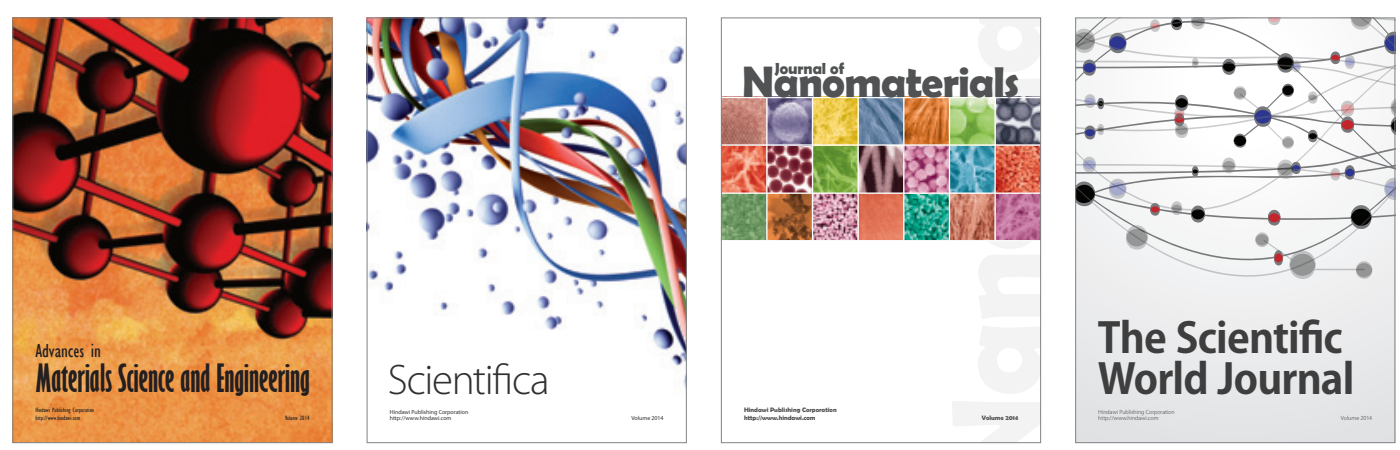

\section{The Scientific World Journal}
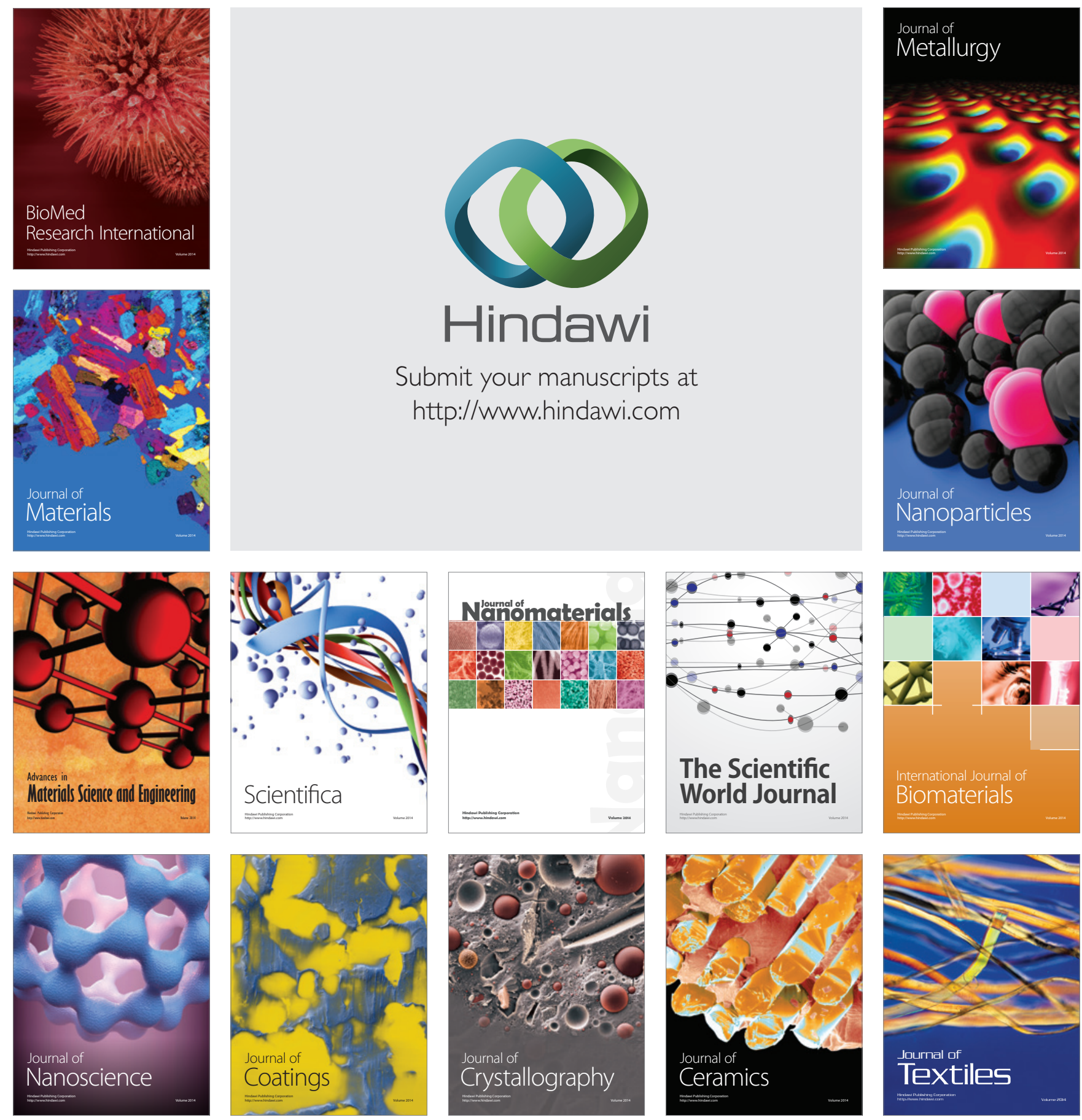